\title{
Biocatalysis for Rare Ginsenoside Rh2 Production in High Level with Co-Immobilized UDP-Glycosyltransferase Bs-YjiC Mutant and Sucrose Synthase AtSuSy
}

\author{
Jianlin Chu ${ }^{1}$, Jiheng Yue ${ }^{2}$, Song Qin ${ }^{1}$, Yuqiang $\mathrm{Li}^{2}$, Bin $\mathrm{Wu}^{2, *}$ and Bingfang $\mathrm{He}^{1, *}$ \\ 1 School of Pharmaceutical Sciences, Nanjing Tech University, No. 30 Puzhu South Road, Jiangbei New Area, \\ Nanjing 211800, China; cj12fl@njtech.edu.cn (J.C.); qinsongtt@njtech.edu.cn (S.Q.) \\ 2 College of Biotechnology and Pharmaceutical Engineering, Nanjing Tech University, No. 30 Puzhu South \\ Road, Jiangbei New Area, Nanjing 211800, China; yuejiheng@163.com (J.Y.); liyuqiang2021@163.com (Y.L.) \\ * Correspondence: wubin1977@njtech.edu.cn (B.W.); bingfanghe@njtech.edu.cn (B.H.); \\ Tel.: +86-025-58139902 (B.W. \& B.H.)
}

Citation: Chu, J.; Yue, J.; Qin, S.; Li,

Y.; Wu, B.; He, B. Biocatalysis for Rare Ginsenoside Rh2 Production in High Level with Co-Immobilized UDP-Glycosyltransferase Bs-YjiC

Mutant and Sucrose Synthase AtSuSy.

Catalysts 2021, 11, 132.

https://doi.org/10.3390/

catal11010132

Received: 16 December 2020

Accepted: 8 January 2021

Published: 18 January 2021

Publisher's Note: MDPI stays neutral with regard to jurisdictional claims in published maps and institutional affiliations.

Copyright: (c) 2021 by the authors. Licensee MDPI, Basel, Switzerland. This article is an open access article distributed under the terms and conditions of the Creative Commons Attribution (CC BY) license (https:// creativecommons.org/licenses/by/ $4.0 /)$
Abstract: Rare ginsenoside Rh2 exhibits diverse pharmacological effects. UDP-glycosyltransferase (UGT) catalyzed glycosylation of protopanaxadiol (PPD) has been of growing interest in recent years. UDP-glycosyltransferase Bs-YjiC coupling sucrose synthase in one-pot reaction was successfully applied to ginsenoside biosynthesis with UDP-glucose regeneration from sucrose and UDP, which formed a green and sustainable approach. In this study, the his-tagged UDP-glycosyltransferase Bs-YjiC mutant M315F and sucrose synthase AtSuSy were co-immobilized on heterofunctional supports. The affinity adsorption significantly improved the capacity of specific binding of the two recombinant enzymes, and the dual enzyme covalently cross-linked by the acetaldehyde groups significantly promoted the binding stability of the immobilized bienzyme, allowing higher substrate concentration by easing substrate inhibition for the coupled reaction. The dual enzyme amount used for ginsenoside Rh2 biosynthesis is Bs-YjiC-M315F: AtSuSy $=18 \mathrm{mU} / \mathrm{mL}: 25.2 \mathrm{mU} / \mathrm{mL}$, a yield of 79.2\% was achieved. The coimmobilized M315F/AtSuSy had good operational stability of repetitive usage for 10 cycles, and the yield of ginsenoside Rh2 was kept between $77.6 \%$ and $81.3 \%$. The high titer of the ginsenoside Rh2 cumulatively reached up to $16.6 \mathrm{mM}(10.3 \mathrm{~g} / \mathrm{L})$ using fedbatch technology, and the final yield was $83.2 \%$. This study has established a green and sustainable approach for the production of ginsenoside $\mathrm{Rh} 2$ in a high level of titer, which provides promising candidates for natural drug research and development.

Keywords: heterofunctional resin; affinity adsorption; UDP-glycosyltransferase mutant; sucrose synthase; bienzyme coimmobilization; ginsenoside Rh2; fed-batch strategy

\section{Introduction}

Ginsenosides are the major bioactive compounds from Chinese medicine Panax ginseng. PPD-type ginsenoside Rh2, an important saponin, showed diverse pharmacological activities, such as anti-oxidation, hepatoprotection, anti-diabetes [1-3], and it is a promising candidate for cancer prevention and therapy [4-7]. Ginsenoside Rh2 content in the natural plant is extremely low (about $0.001 \%$ in dried Panax ginseng roots) [8]. Ginsenosides are biosynthesized by glycosylation of protopanaxadiol (PPD) and (protopanaxatriol) PPT [9]. UDP-glycosyltransferases (UGTs), such as PgUGT74AE2, UGTPg45, and UGTPg29 from P. ginseng [10,11], are responsible for PPD-type and PPT-type ginsenosides (Rh2, CK, Rh2, F2, and Rh1) production [10-12]. UGTPg45-HV mutant involved yeast cell factory adopted to produce ginsenoside Rh2 with the titer of $179.3 \mathrm{mg} / \mathrm{L}$ in a shake flask system and $2.25 \mathrm{~g} / \mathrm{L}$ in a $10 \mathrm{~L}$ fed-batch fermentation [13]. Using the stoichiometric quantities of highcost substrate UDPG as sugar donor, UGT73F3 from Medicago truncatula, UGT73C10 from Barbarea vulgaris, UGT73C5 from Arabidopsis thaliana and UGTPn50 from P. ginseng could 
catalyze the C3-OH glycosylation of PPD to produce Rh2 [14-16]. Additionally, UGT73C5 from $A$. thaliana was able to selectively glycosylate the $\mathrm{C} 3-\mathrm{OH}$ of PPD and ginsenoside CK to form ginsenosides Rh2 and F12, and $3.2 \mathrm{~g} / \mathrm{L}$ ginsenoside $\mathrm{Rh} 2$ with a yield of $87 \%$ was attained by UGT73C5-AtSuSy coupled reactions [14].

Microbial UGTs often show substrate flexibility and are potential candidates for ginsenoside Rh2 production. Recently, several microbial UGTs (UGT51 from Saccharomyces cerevisiae, UGT109A1 and Bs-YjiC from Bacillus subtilis 168) demonstrated the glycosylation of PPD or PPT [17-19]. UGT51 mutant in yeast cell factories could biosynthesize Rh2 up to $300 \mathrm{mg} / \mathrm{L}$ [17]. Bs-YjiC or UGT109A1 could catalyze the C3-OH and C12-OH of PPD glycosylation to generate ginsenoside $\mathrm{Rh} 2$ and unnatural ginsenoside F12 [19]. Using $B s-Y j i C$ and sucrose synthase AtSuSy coupled one-pot reaction for UDP-glucose regeneration from sucrose and UDP, ginsenoside F12 $(3.98 \mathrm{~g} / \mathrm{L})$ and ginsenoside Rh2 $(0.20 \mathrm{~g} / \mathrm{L})$ was obtained [19]. Bs-YjiC may show an important application for novel ginsenosides synthesis, and five PPT-type ginsenosides, including ginsenoside Rh1 and four unnatural ginsenosides, were produced [20]. In our previous report, the regiospecificity of Bs-YjiC toward ginsenoside Rh2 biosynthesis was investigated by semi-directed evolution [21]. Bs-YjiC mutant M315F showed the highest regioselectivity of $99 \%$ for ginsenoside $\mathrm{Rh} 2$ formation (conversion $\sim 82 \%$ ). By Bs-YjiC mutant M315F-AtSuSy coupled one-pot reaction, an extraordinarily high yield of Rh2 (3.7 g/L) was acquired by periodic feeding of PPD [21].

Enzymes immobilized onto supports may be feasible for desired products using batch or continuous process, together with improving operational stability [22]. It could facilitate the recyclability of biocatalyst from the bioreaction medium, showing the benefits of convenient handling of the enzyme, facile separation from reaction media [23]. The coimmobilized enzymes of glucose oxidase (GOX) and horseradish peroxidase (HRP) on the surface of silica microparticles showed higher overall enzymatic activity when compared to the enzymes in separated layers and free enzymes [24]. As the supports with metal chelate groups, four of six co-ordinations of $\mathrm{Ni}^{2+}$ ions were formed by the four ligands of the NTA, while the spare two positions were occupied by other molecules, and this could be selectively replaced by the His tag included in the sequence of biomolecules [25,26]. Thus, immobilization of recombinant enzymes with polyhistidine linkers could accurately capture the specific binding functional groups (typically nitrilotriacetic acid, NTA) on the carriers $[25,26]$. It may be easy to control the different ratio of multi-enzyme catalyzed cascade with improved catalytic efficiency. Seven his-tagged enzymes involving the biosynthesis of uridine diphosphate galactose (UDP-galactose) were coimmobilized through $6^{*}$ his tags onto nickel agarose beads [27], and the biocatalyzed production of UDP-galactose by the co-immobilized multi-enzymes was more efficient than that by free enzymes [27]. Biocatalysts immobilization through specific binding could regulate the original orientation of the enzymes without unfavorable changes by non-specific interactions with the carrier surface. It may also impact the ability to co-localize multiple enzymes in close proximity of the active sites to one another. There is no report involving co-immobilization of glycosyltransferase mutant and sucrose synthase to recycle the two biocatalysts from the reaction media for the production of Rh2.

In order to achieve a higher level of product Rh2 (Scheme 1), this study applied the coimmobilization of glycosyltransferase Bs-YjiC mutant M315F and sucrose synthase AtSuSy onto heterofunctional resin. His-tagged bienzyme co-immobilizing with two-step of affinity adsorption and covalently cross-linking the acetaldehyde groups was applied. The properties of co-immobilized enzymes were investigated, and the catalytic yield for $\mathrm{Rh} 2$ biosynthesis and operational stability by the co-immobilized enzymes were also studied. The cumulative production of Rh2 in a high level by biotechnological fed-batch feeding was also adopted using the co-immobilized enzymes. 


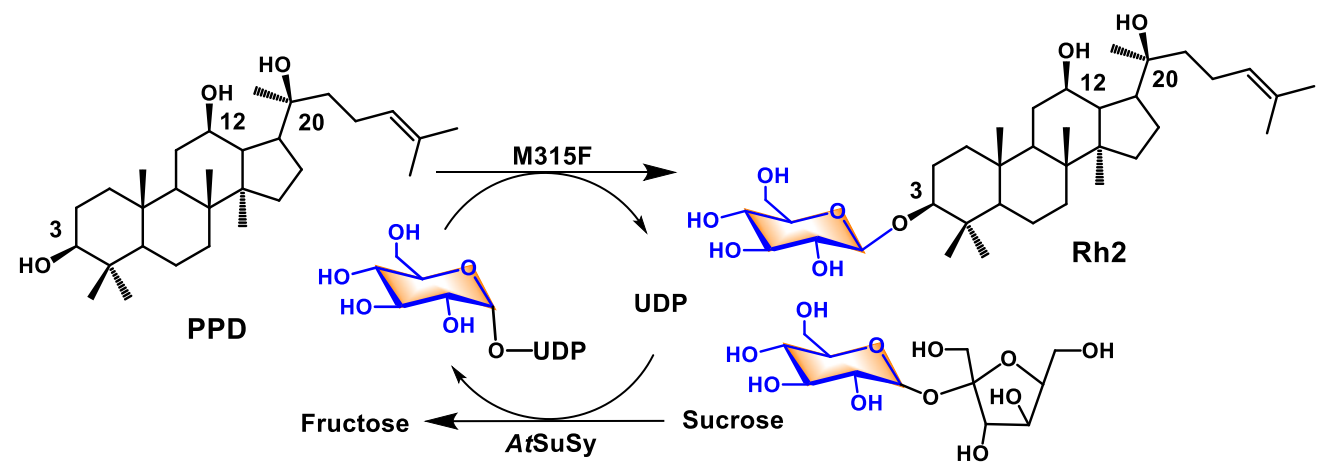

Scheme 1. One-pot reaction catalyzed by M315F and AtSuSy for biosynthesis of Rh2 coupled with UDPG regeneration.

\section{Results and Discussion}

\subsection{Co-Immobilization of Dual Enzyme onto Heterofunctional Carriers}

Macroporous resin LX1000HG was selected as the support for modification and bienzyme adsorption. Resin LX1000HG modified with two kinds of functional groups was prepared for the dual enzyme co-immobilization. Heterofunctional support with glyoxyl-metal-chelate bifunctional groups was inspired for his-tagged enzyme immobilization [26-28]. The two-step immobilization was firstly finished as the metal-chelate groups were able to promote the specific adsorption of the proteins, and then glyoxyl groups enabled the adsorbed enzyme to covalently anchor to the resin (Scheme 2). The structural flexibility of the immobilized enzymes was critical for substrate binding and its catalytic function [27]. Glyoxyl-metal-chelate supports involved coordination bonding with $\mathrm{Ni}^{2+}, \mathrm{Cu}^{2+}$, and $\mathrm{Co}^{2+}$ were respectively applied for individual adsorption of his-tagged M315F and AtSuSy. In the case of the $\mathrm{Ni}^{2+}$-charged support, the highest activity expression, recovery, and specific activity were observed by both M315F and AtSuSy (Table 1). Another kind of heterofunctional resin, amino-glyoxyl resin, was also used for M315F and AtSuSy adsorption. No further improved results were obtained (data not shown).

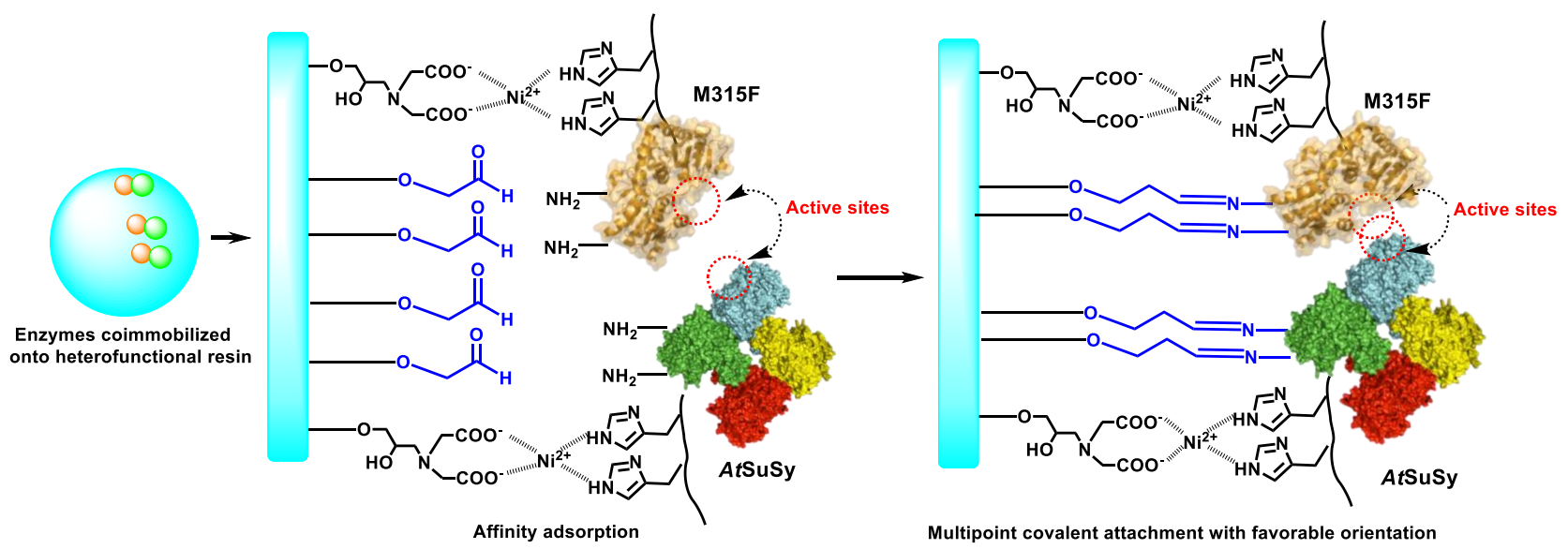

Scheme 2. Absorption procedures for two-step immobilization of his-tagged M315F and AtSuSy.

For the M315F adsorption by heterofunctional resin, the expressed activity was $718.5 \mathrm{mU} / \mathrm{g}$ wet support as the collaborative coordination bonding of the His tag of M315F and IDA to the $\mathrm{Ni}^{2+}$. The specific activity of the M315F binding on glyoxyl- $\mathrm{Ni}^{2+}$-chelate carriers was $412.7 \mathrm{mU} / \mathrm{mg}$ bound protein, and it produced the recovery of $47.9 \%$ and enzyme loading of $1.74 \mathrm{mg} / \mathrm{g}$ wet carriers (Table 1 ). The enzyme activity expression by the specific bound AtSuSy onto glyoxyl-Ni ${ }^{2+}$-chelate resin was $1959.5 \mathrm{mU} / \mathrm{g}$ wet support, and the specific activity of the adsorbed AtSuSy was $1660.5 \mathrm{mU} / \mathrm{mg}$ bound protein, resulting in the recovery of $39.1 \%$ and enzyme loading of $1.18 \mathrm{mg} / \mathrm{g}$ wet carriers (Table 1 ). 
Table 1. Heterofunctional support on the effect of adsorbed individual M315F and AtSuSy.

\begin{tabular}{|c|c|c|c|c|c|}
\hline Enzyme & Heterofunctional Support & $\begin{array}{c}\text { Activity } \\
\text { (mU/g Wet Support) }\end{array}$ & $\begin{array}{c}\text { Enzyme Activity Recovery } \\
\text { (\%) }\end{array}$ & $\begin{array}{l}\text { Protein Adsorption } \\
\text { (mg/g Wet Support) }\end{array}$ & $\begin{array}{l}\text { Specific Activity of } \\
\text { Immobilized Enzyme } \\
\text { (U/mg Protein) }\end{array}$ \\
\hline \multirow{3}{*}{ M315F } & Glyoxyl-Ni ${ }^{2+}$-chelate resin & 718.5 & $47.9 \%$ & 1.74 & 412.7 \\
\hline & Glyoxyl- $\mathrm{Cu}^{2+}$-chelate resin & 182.1 & $12.1 \%$ & 2.54 & 71.7 \\
\hline & Glyoxyl- $\mathrm{Co}^{2+}$-chelate resin & 629.0 & $41.9 \%$ & 2.71 & 232.1 \\
\hline \multirow{3}{*}{ AtSuSy } & Glyoxyl-Ni ${ }^{2+}$-chelate resin & 1959.5 & $39.1 \%$ & 1.18 & 1660.5 \\
\hline & Glyoxyl- $\mathrm{Cu}^{2+}$-chelate resin & 1862.5 & $37.2 \%$ & 2.46 & 350.6 \\
\hline & Glyoxyl- $\mathrm{Co}^{2+}$-chelate resin & 1636.5 & $32.7 \%$ & 1.71 & 957.0 \\
\hline
\end{tabular}

Moreover, the conditions for enzyme adsorption were also optimized, the temperature of $25^{\circ} \mathrm{C}, \mathrm{pH} 7.0$, and adsorption time of $6 \mathrm{~h}$ (Figure S1) was selected for the his-tagged $\mathrm{M} 315 \mathrm{~F}$ and AtSuSy co-binding onto heterofunctional resin. For the one-pot reaction of PPD glycosylating by coupling M315F to AtSuSy from sucrose and UDP, the portion of the co-immobilized dual enzyme was critical for its catalytic activities. The portion of M315F and AtSuSy after co-immobilization was regulated by the initial addition of two enzymes in different amounts, and the optimized portion of co-immobilized M315F / AtSuSy onto the resin at the activity ratio of 1:1.4 was selected for effective production of Rh2, which reached $69.3 \%$ conversion at high substrate PPD $(6 \mathrm{mM})$ and high regioselectivity $(99 \%)$ for Rh2 formation (Figure 1A). Free enzymes M315F-AtSuSy displayed a conversion of $\sim 60 \%$ at $2 \mathrm{mM}$ PPD in our previous study [21], suggesting the inhibition on the catalytic yield under high PPD concentration, and similar substrate inhibition was also revealed by the UGT73C5-SuSy coupled reaction [14]. The dual enzyme ratio after coimmobilization for the M315F-AtSuSy coupled reaction was much lower than that of 1:2.1 in our previous report [21]. Then, the bound M315F and AtSuSy were further conducted by the covalent cross-linking immobilization between the glyoxyl groups and adsorbed enzymes. The recovery of $50.9 \%$ by M315F and $48.3 \%$ by AtSuSy was resulted from the above adsorption optimization and covalent cross-linking coimmobilization. After coimmobilization, the co-localized multiple enzymes in close proximity of the active sites to one another with a suitable orientation by first step specifical adsorption may be beneficial to control the covalent cross-linking in the next step, which leads to high activity expression, facilitating the access of substrates between one enzyme and another in an adequate concentration to perform improved reaction [27].
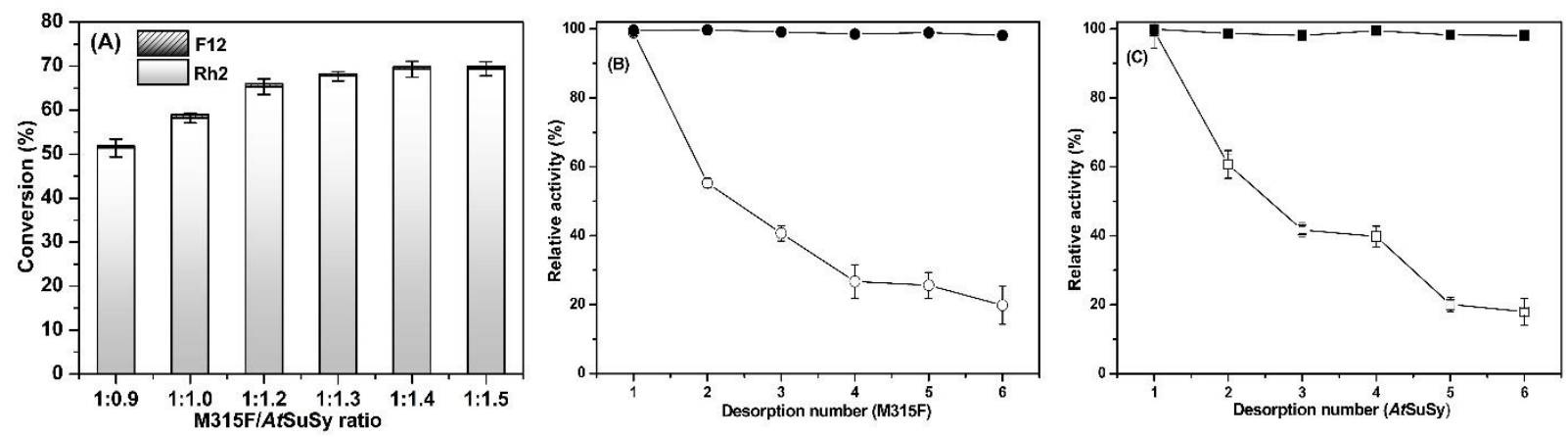

Figure 1. (A) Effect of the ratio of co-immobilized bienzyme M315F/AtSuSy for Rh2 production. The yield of products Rh2 and F12 was recorded. (B) Desorption of immobilized M315F, his-tagged M315F directly specifical binding with $\mathrm{Ni}^{2+}$-chelate groups $(\bigcirc)$, co-immobilized M315F by affinity adsorption and covalently cross-linking $(\bullet)$. (C) Desorption of co-immobilized AtSuSy, his-tagged M315F directly specifical binding with $\mathrm{Ni}^{2+}$-chelate groups ( $\square$ ), co-immobilized M315F by affinity adsorption and covalently cross-linking ( $\boldsymbol{\square})$. Enzyme desorption was conducted by measuring the enzyme activity in supernatant and suspension with $0.5 \mathrm{M} \mathrm{NaCl}$ solution ( $\mathrm{pH} 7.0$ ), the suspension was maintained under very gentle stirring for $60 \mathrm{~min}$. 
The binding stability of the immobilized enzymes was investigated (Figure 1). The M315F and AtSuSy directly adsorbed by specifical binding with $\mathrm{Ni}^{2+}$-chelate groups were easy to lose the total enzyme activity using buffer wash over twice, which both M315F and AtSuSy retained less than $60 \%$ of activities (Figure 1B,C) by desorption test, suggesting the enzymes through direct affinity adsorption were easy to leach from the resin and showed the low ability to bear mild conditions. The M315F and AtSuSy onto glyoxyl$\mathrm{Ni}^{2+}$-chelate supports by two-step of co-immobilization retained high activities (nearly 99\%) after seven times of desorption (Figure 1B,C), nearly no enzyme activity was leached. Most importantly, the co-immobilization of M315F and AtSuSy with the two-step ways significantly improved the binding stability of the co-immobilized bi-enzyme, though histagged enzymes specifical binding with $\mathrm{Ni}^{2+}$-chelate groups and covalently cross-linking between the glyoxyl groups and the adsorbed proteins [29].

\subsection{Physicochemical Properties of Heterofunctional Carriers}

The SEM images of bifunctional macroporous resin revealed that the surface of supports was smooth and uniform with some large holes on the surface (Figure 2A), and the internal morphology of the resin also showed the shape of various pore volumes (Figure 2B,C). After enzyme co-immobilization, pores on the surface of resin were coarse and rough as covered with many enzyme particles, meaning that the out surface of carriers had been utilized for the enzyme adsorption (Figure 2D). Moreover, the internal morphologies of resin showed that it contained various smaller spores as the binding of the enzymes (Figure 2E,F).
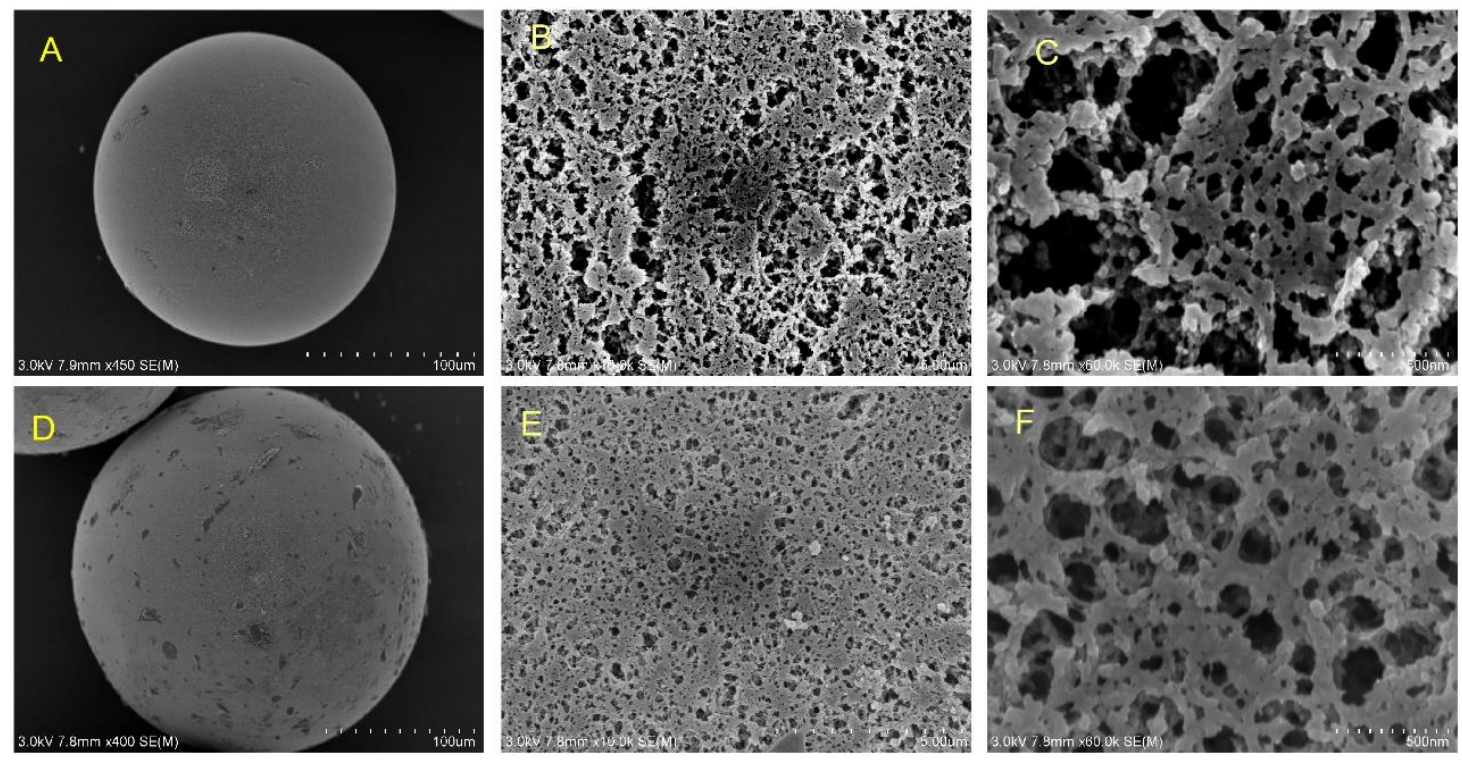

Figure 2. SEM images of resin heterofunctional resin (A-C) and resin immobilized with M315F/AtSuSy (D-F).

The carriers and dual enzymes co-immobilized onto resin were analyzed by FT-IR, the results can be found in Figure S2. The successful dual enzyme immobilization was further confirmed by the measurements of specific surface and void analyzer. Compared with heterofunctional resin, the carriers adsorbed with enzymes showed a decrease in the pore volumes (from 0.47 to $0.37 \mathrm{~cm}^{3} / \mathrm{g}$ ) (Table 2). The pore volume decrease may be attributed to the enzyme occupation in the pore channels, suggesting that enzymes had been immobilized on resin [30], while the specific surface area increased from 41.34 to $58.93 \mathrm{~m}^{2} / \mathrm{g}$ (Table 2) as the enzymes immobilized onto the support. The average pore diameter of heterofunctional resin was $33.7 \mathrm{~nm}$, which matched the requirement of pore diameter for the UGTs and AtSuSy binding to prevent restrictions of the enzyme access [31]. After immobilization, the average pore diameter of supports dropped to $27.6 \mathrm{~nm}$, suggesting resin was successfully binding with enzymes. 
Table 2. Textural properties derived from $\mathrm{N}_{2}$ adsorption of heterofunctional resin and support with derivatives.

\begin{tabular}{|c|c|c|c|}
\hline Support & $\begin{array}{c}\text { Specific } \\
\text { Surface Area } \\
\left(\mathrm{m}^{2} / \mathrm{g}\right)\end{array}$ & $\begin{array}{l}\text { Pore Volume } \\
\qquad\left(\mathrm{cm}^{3} / \mathrm{g}\right)\end{array}$ & $\begin{array}{l}\text { Average Pore } \\
\text { Diameter } \\
\text { (nm) }\end{array}$ \\
\hline Heterofunctional LX1000HG & 41.34 & 0.47 & 33.67 \\
\hline Resin with co-immobilized enzymes & 58.93 & 0.37 & 27.64 \\
\hline
\end{tabular}

\subsection{Effect of $\mathrm{pH}$ and Temperature on Activity and Stability of Enzymes}

Both free M315F and co-immobilized M315F had the highest activity at $\mathrm{pH} 8.0$ (Figure S3A). Free sucrose synthase AtSuSy had the highest activity at pH 7.0, and coimmobilized AtSuSy showed the highest activity at pH 6.0. Both free AtSuSy and coimmobilized AtSuSy were most active under the $\mathrm{pH}$ range from 6.0 to 8.0, which showed 90\% activity (Figure S3B). After co-immobilization, both enzyme M315F and AtSuSy exhibited $\mathrm{pH}$ stability improvement (Figure S4). The immobilized AtSuSy was stable under the $\mathrm{pH}$ range from 6.0 to 9.0 , retaining $90 \%$ initial activities. The immobilized AtSuSy still kept nearly $80 \%$ of initial activity at $\mathrm{pH} 10.0$ for $12 \mathrm{~h}$ incubation. The immobilized M315F displayed improved $\mathrm{pH}$ stability as it maintained $90 \%$ initial activities $(12 \mathrm{~h}$ ) at a $\mathrm{pH}$ range from 6.0 to 9.0 (Figure S4).

Both free and immobilized AtSuSy had the highest activity at $40{ }^{\circ} \mathrm{C}$ and were showed high activities at a temperature range from 35 to $40^{\circ} \mathrm{C}$ (Figure S5). Free enzyme M315F had the highest activity at $35^{\circ} \mathrm{C}$ (Figure S5), while the immobilized M315F were most active at $40^{\circ} \mathrm{C}$, and also showed high activity at a temperature range from 35 to $45^{\circ} \mathrm{C}$. The immobilized enzyme M315F showed the improvement of thermal stability. The immobilized enzyme M315F possessed good thermal stability below $35^{\circ} \mathrm{C}$ as it retained nearly $100 \%$ initial activity. However, free M315F just retained $<70 \%$ initial activity when incubation at $35^{\circ} \mathrm{C}$ for $12 \mathrm{~h}$ (Figure $3 \mathrm{~A}$ ). There is a certain improvement of thermal stability by the immobilized enzyme of AtSuSy. The co-immobilized AtSuSy was stable when incubated under the temperature below $35^{\circ} \mathrm{C}$ for $12 \mathrm{~h}$, and it retained nearly $100 \%$ initial activity, while free AtSuSy just showed about $80 \%$ activity at $35^{\circ} \mathrm{C}$ (Figure 3B). Such improved properties of co-immobilized AtSuSy and M315F were beneficial to the cascade reaction for the ginsenoside Rh2 production.
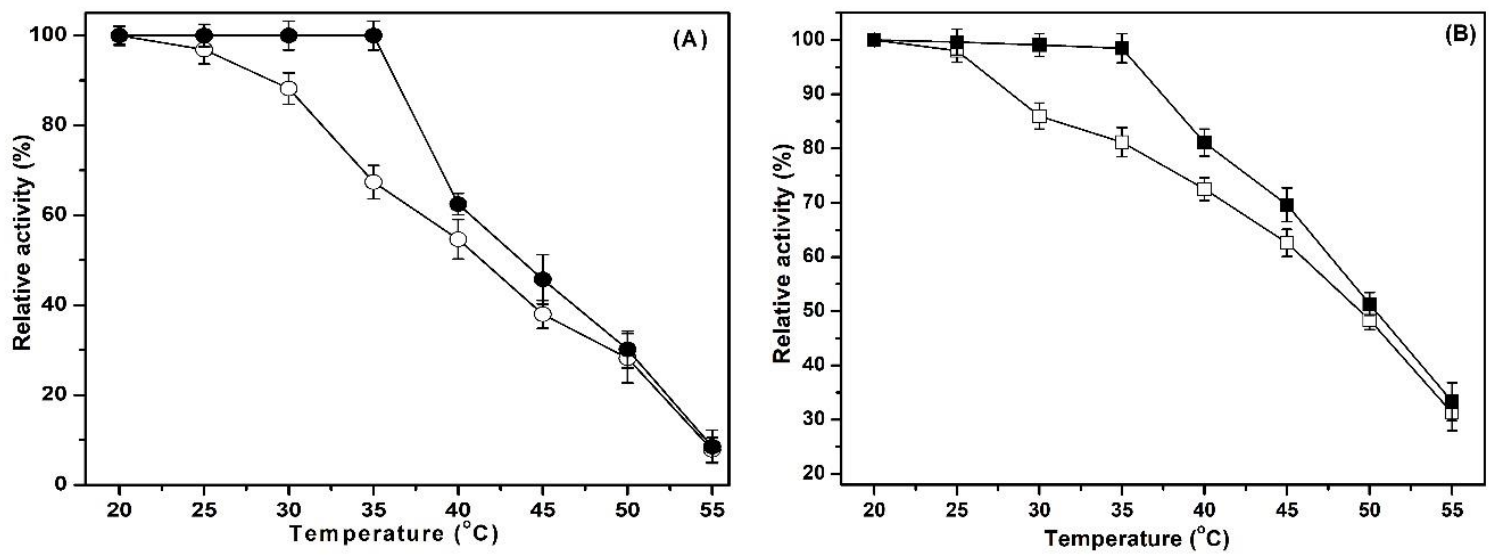

Figure 3. Thermal stability comparison of free and co-immobilized M315F (A), thermal stability comparaison of free and AtSuSy (B).

\subsection{Conditions for the Co-Immobilized Enzymes Catalyzed Reactions}

AtSuSy could biosynthesize UDPG using sucrose as the expedient sugar donor $[13,14,19,21]$, and the UGTs-AtSuSy coupled one-pot reaction are a useful protocol for glycosides production with continuous UDPG regeneration [14,19]. In our previous report, Bs-YjiC mutant M315F- 
AtSuSy coupled one-pot reaction was applied for UDPG regeneration from UDP and cheap sucrose, and it was demonstrated to produce ginsenoside Rh2 up to $3.7 \mathrm{~g} / \mathrm{L}$ using PPD as substrate [21]. The reaction conditions by the co-immobilized enzymes for the biosynthesis of ginsenoside $\mathrm{Rh} 2$, such as $\mathrm{pH} 8.0$, temperature $35^{\circ} \mathrm{C}$, and $0.4 \mathrm{M}$ sucrose and $5 \mathrm{mM} \mathrm{Mg}{ }^{2+}$ were generally the same as in our previous report [21].

For the co-immobilized dual enzyme M315F and AtSuSy catalyzed cascade reaction, the other conditions were further optimized to reach a higher level of $\mathrm{Rh} 2$ production. Our previous study disclosed that the conversion by free M315F-AtSuSy coupled reaction was about $60 \%$ using $2 \mathrm{mM}$ substrate PPD [21]. The concentration of PPD showed a significant impact on the conversion for ginsenoside $\mathrm{Rh} 2$ production by the co-immobilized enzymes (Figure 4A). Using $2 \mathrm{mM}$ PPD as substrate, it showed a higher conversion $(\sim 99 \%)$ by coimmobilized M315F and AtSuSy than that by free enzymes, indicating the co-immobilized bienzyme could improve the conversion rate under higher PPD concentration and allowing higher substrate concentration by easing substrate inhibition for the coupled reaction $[24,27]$. The co-immobilized enzymes catalyzed reaction showed the decreased conversion from $99 \%$ to $50 \%$ as the addition of PPD concentration increased from 2 to $10 \mathrm{mM}$, and the co-immobilized enzymes displayed higher activity than that of free enzymes at high PPD substrate concentration [22,23]. Interestingly, the titer of produced ginsenoside $\mathrm{Rh} 2$ was gradually raised as the PPD concentration increased. As a result, about $5.05 \mathrm{mM}$ ginsenoside Rh2 (conversion 63.1\%) was obtained using an initial concentration of $8 \mathrm{mM}$ PPD (Figure 4A), the titer of ginsenoside Rh2 by the co-immobilized dual enzyme was higher than that by free enzymes. A higher concentration of PPD (above $10 \mathrm{mM}$ ) may not be beneficial for the reaction. Thus, an initial PPD concentration of $8 \mathrm{mM}$ was selected for the following optimization of reaction conditions by co-immobilized enzymes.
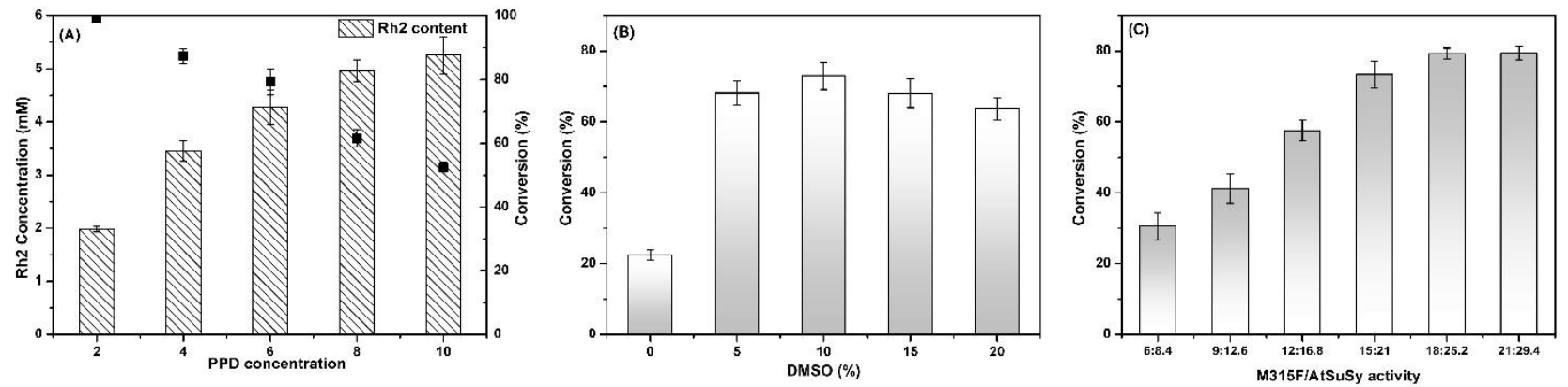

Figure 4. Optimization of the conditions for co-immobilized M315F and AtSuSy coupled reactions. Effect of PPD concentration on the Rh2 production by co-immobilized M315F and AtSuSy catalyzed reactions (A), conversion yield ( $\mathbf{\square})$ was recorded. Effect of co-solvent DMSO on the yield of Rh2 by co-immobilized M315F and AtSuSy catalyzed reactions (B), and influence of different co-immobilized M315F and AtSuSy usage on the glycosylation reaction for Rh2 formation (C).

The addition of hydrophilic co-solvent DMSO could show the effect of substrate PPD solubility increasing in the reaction media, resulting in a higher PPD initial concentration [14]. The conversion (above 60\%) by co-immobilized enzymes in the hydrophilic media contained $5 \%-20 \%(v / v)$ DMSO was higher than that in the buffer system (yield $22.4 \%$ ) (Figure 4B). The conversion dramatically increased as the reaction media with the addition of $5 \%(v / v)$ DMSO and $10 \%(v / v)$ DMSO. The highest conversion was $72 \%$ using $10 \%(v / v)$ DMSO as the reaction media, which was about three times the yield by using buffer media. The yield slightly decreased using 15\% $(v / v)$ DMSO (yield 68.1\%) and $20 \%(v / v)$ DMSO (yield 63.7\%) as the reaction media, suggesting the drawback of activity inhibition by higher a content of DMSO addition. There was no significant influence on the yield of ginsenoside Rh2 by increasing the addition of UDP, only a catalytic amount of UDP $(0.5 \mathrm{mM})$ was necessary for the co-immobilized M315F-AtSuSy coupled reaction (Figure S6). The conversion was greatly improved with the increased addition of coimmobilized biocatalysts. Using the co-immobilized enzymes at Bs-YjiC-M315F: 
AtSuSy $=18 \mathrm{mU} / \mathrm{mL}: 25.2 \mathrm{mU} / \mathrm{mL}$, a high yield of $79.2 \%$ was achieved (Figure $4 \mathrm{C}$ ). The biocatalyst usage for ginsenoside $\mathrm{Rh} 2$ production was much lower than that reported in other studies [14,19].

\subsection{Reusability of Co-Immobilized Enzymes and Fed-Batch Strategy for Ginsenoside Rh2}

The co-immobilized glycosyltransferase Bs-YjiC mutant M315F and sucrose synthase AtSuSy exhibited good operational stability after repetitive 10 cycles of dual enzyme coupled reaction for ginsenoside Rh2 production. The co-immobilized sucrose synthase AtSuSy could maintain high catalytic activities of UDPG regeneration using sucrose and UDP, and co-immobilized M315F equally kept high activity of ginsenoside Rh2 synthesis, yielding a total conversion between $77.6 \%$ and $81.3 \%$ (Figure $5 \mathrm{~A}$ ) during the 10 consecutive cycles usage. This implied the good operation stability of the M315F-AtSuSy co-immobilized onto heterofunctional resin.
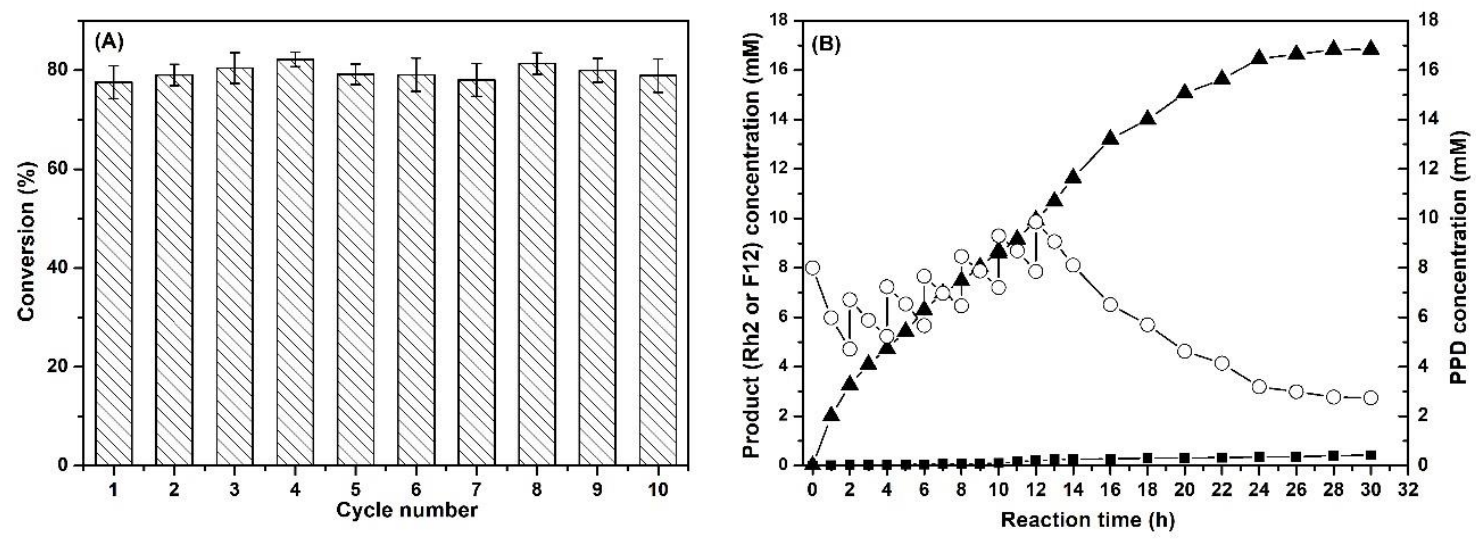

Figure 5. Reusability of the co-immobilized M315F and AtSuSy for Rh2 production (A). Fed-batch biosynthesis of ginsenoside Rh2 by co-immobilized M315F and AtSuSy coupled reaction (B), and PPD ( $\bigcirc)$, product of ginsenoside Rh2 ( $\mathbf{\Lambda}$ ), product of ginsenoside F12 (ם) was measured.

To further alleviate the possible inhibition by high substrate concentration, a fedbatch strategy by stepwise addition of $2.0 \mathrm{mM}$ fresh PPD was adopted to the cascade reaction with the co-immobilized M315F-AtSuSy. The initial concentration of PPD (8 mM) was used, a titer of the ginsenoside Rh2 cumulatively reached up to $16.6 \mathrm{mM}(10.3 \mathrm{~g} / \mathrm{L})$ (Figure 5B) by using fed-batch technology and the final yield was about $83.2 \%$. To our acknowledgment, this titer of ginsenoside $\mathrm{Rh} 2$ produced by the present biotechnological way was the highest level, and it was 2.78-3.2 times of free enzymes [21], and much higher than other reports [14]. Using co-immobilized M315F-AtSuSy coupled reaction with fedbatch protocol, the overall STY of ginsenoside Rh2 was about $0.396 \mathrm{~g} / \mathrm{L} / \mathrm{h}$, which was 20 -fold higher than that of the report [13]. The co-immobilized M315F-AtSuSy catalyzed the transglycosylating reaction afforded a new way for efficient manufacturing of rare ginsenoside Rh2, and formed a green, cost-effective and sustainable approach with UDPglucose regeneration from sucrose and catalytic amount of UDP.

\section{Materials and Methods}

\subsection{Materials and Chemicals}

The C-terminal 6*his-tagged glycosyltransferase Bs-YjiC (from Bacillus subtilis 168) mutant M315F and C-terminal 6*his-tagged sucrose synthase AtSuSy (from Arabidopsis thaliana) were obtained and prepared respectively as in our previous report [21]. Macroporous resin LX1000HG (contained hydroxyl group) was from Sunresin New Materials Co., Ltd. (Xi'an, China). PPD, Rh2, and F12 were purchased from Nanjing Spring \& Autumn Biological Engineering Co., Ltd. (Nanjing, China). Uridine 5'-diphosphate glucose (UDPG) and uridine $5^{\prime}$-diphosphate sodium salt (UDP) were obtained from Nanjing Duly Biotech Co., Ltd. (Nanjing, China). Sucrose, epichlorohydrin, iminodiacetic acid (IDA), 
and other chemicals used were all analytical grade. Solvents used for high-performance liquid chromatography (HPLC) analysis were of HPLC grade. Bradford Protein Assay Kit and bovine serum albumin for Protein Concentration Assay were from Sangon Biotech (Shanghai, China).

\subsection{Resin Modified with Bifunctional Groups and Carries Characterization}

Macroporous resin LX1000HG (contained hydroxyl group) modification was according to ref. [29,32], and the procedure was also shown in Figure S7. It was firstly modified with epichlorohydrin to obtain epoxy-activated resin LX1000HG. Epoxy-resin LX1000HG was used in the treatment with iminodiacetic acid (IDA), then produced diol (glyceryl) groups and IDA groups activated resin. The support was washed with distilled water, and further oxidation of glyceryl groups was achieved using $100 \mathrm{~mL}$ of sodium periodate $\left(\mathrm{NaIO}_{4}\right)$, producing glyoxyl-IDA-activated resin [29,32]. To completely block the epoxy group, the resin was incubated with $3.0 \mathrm{M}$ glycine for $16 \mathrm{~h}$ at $20^{\circ} \mathrm{C}$ [33]. GlyoxylIDA-activated resin was added to the metal chelate solutions $\left(30 \mathrm{mg} / \mathrm{mL}, \mathrm{CoCl}_{2} \cdot 6 \mathrm{H}_{2} \mathrm{O}\right.$, $\mathrm{NiCl}_{2} \cdot 6 \mathrm{H}_{2} \mathrm{O}$, or $\mathrm{CuSO}_{4} \cdot 5 \mathrm{H}_{2} \mathrm{O}$ ) at $\mathrm{pH} 7.0$ for $1.0 \mathrm{~h}$ at $25^{\circ} \mathrm{C}$ [28]. Finally, the glyoxyl-IDA metal chelate bifunctionalized resin was washed with distilled water and vacuum dried for enzyme adsorption.

The morphology of bifunctional resin and support with co-immobilized enzymes of M315F-AtSuSy were examined by a scanning electron microscope (SEM, S-4800, Hitachi, Tokyo, Japan). During imaging, the scanning electron microscope had an acceleration voltage of $3.0 \mathrm{kV}$. The samples were freeze-dried and prepared for microscope imaging.

The FTIR spectra were obtained on samples in KBr pellets using a Shimadzu FTIR$8400 S$ (Tokyo, Japan) spectrometer in the frequency range of 400 to $4000 \mathrm{~cm}^{-1}$. The samples were mixed with $1 \%(w / w) \mathrm{KBr}$, and the analysis was employed at 10 scans per second with a resolution of $4 \mathrm{~cm}^{-1}$.

The specific surface area, pore volumes, and average pore diameters of resin and support with co-immobilized enzymes were measured by obtaining $\mathrm{N}_{2}$ adsorption-desorption (ASAP 2020 V4.00, Micromeritics Instrument Co., Ltd., Shanghai). The specific areas were estimated by the standard Brunauer-Emmett-Teller (BET) method, and the distributions of pore diameters were estimated by the desorption branches of the isotherms with the Barrett-Joyner-Halenda (BJH) model.

\subsection{Dual Enzyme Co-Immobilization with Adsorption and Covalent Attachment}

E. coli BL21(DE3) crude extract was used as a multi-protein model system during the immobilization tests with different supports. For the co-immobilization of engineered M315F and AtSuSy onto heterofunctional resin with two kinds of groups, the two-step immobilization protocol was accomplished according to Mateo et al. [32,33]. His tag affinity method is generic and can be easily applied to specific adsorption $[28,34]$. The crude two enzymes from E. coli BL21(DE3) crude cell extract (M315/AtSuSy total activity addition of $4000 \mathrm{mU} / \mathrm{g}$ wet resin) were in $10 \mathrm{~mL}$ solution $(25 \mathrm{mM}$ Tris- $\mathrm{HCl}$ buffer, $\mathrm{pH} 7.0)$ at $25^{\circ} \mathrm{C}$ and adsorbed by heterofunctional resin ( $1.0 \mathrm{~g}$ wet resin) under mild stirring for $6 \mathrm{~h}$. In the first step, the optimal ratio of M315F/AtSuSy adsorbed by resin for the biosynthesis of Rh2 was determined using the various ratios of M315F/AtSuSy adjustment, next, adsorption time, $\mathrm{pH}$, and temperature were studied, respectively.

The final step was incubated with $50 \mathrm{mM}$ Gly-NaOH buffer $\mathrm{pH} 10.0$ for $3 \mathrm{~h}$ (intermittent shaking) to allow an intramolecular multipoint covalent attachment between the glyoxyl groups on the support and the amino/other groups on the enzyme surface. Finally, the immobilized enzyme was washed with excess distilled water. The resin with co-immobilized enzymes was separated, and samples of the supernatants and resins were withdrawn. The enzyme activity was measured and the protein concentration of the solution was also analyzed. The biocatalyst was stored at $4{ }^{\circ} \mathrm{C}$ until further use. 
The immobilization yield $\mathrm{IY}_{\mathrm{imob}}(\%)$ was calculated via the difference between the activity of the free enzyme solution $\left(\mathrm{A}_{\mathrm{tf}}\right)$ and the remaining activity of the supernatant $\left(\mathrm{A}_{\mathrm{ts}}\right)$ using Equation (1):

$$
\operatorname{IY} Y_{\text {imob }}(\%)=\left(A_{t f}-A_{t s}\right) / A_{t f} \times 100 \%
$$

The amount of enzyme offered for each gram of resin $\left(A t_{\text {off }}-U / g\right)$ and the immobilization yield were individually collected to calculate the theoretical activity $\left(\mathrm{At}_{\mathrm{t}}\right)$ of immobilized enzyme M315F or AtSuSy, as shown in Equation (2):

$$
A t_{t}=A t_{\text {off }} \times I Y
$$

The co-immobilized enzymes activity $\left(A t_{d}\right)$ individually assayed by the theoretical activity $\left(A t_{t}\right)$ in the solution was defined as the enzymatic activity recovery according to Equation (3):

$$
A t_{r}=A t_{d} / A t_{t} \times 100 \%
$$

\subsection{Enzyme Activity and Protein Assay}

The enzyme activities of glycosyltransferase Bs-YjiC mutant M315F and sucrose synthase AtSuSy were measured as in our previous report [21]. Protein concentration in solution was examined by Bradford's method using bovine serum albumin as Ref. [35].

\subsection{Biochemical Properties of Free and Co-Immobilized Enzymes}

For the characterization of free and co-immobilized M315F and AtSuSy, the effects of $\mathrm{pH}$ on the activity and $\mathrm{pH}$ stability $\left(35^{\circ} \mathrm{C}, 12 \mathrm{~h}\right)$ of enzymes were determined in various buffer systems ( $\mathrm{pH}$ 5.0-10.0). The effects of temperature on the activity and thermal stability $(\mathrm{pH} 8.0,12 \mathrm{~h}$ ) of free and immobilized enzymes were determined at different temperatures (from 20 to $55^{\circ} \mathrm{C}$ ).

\subsection{Optimization of the Co-Immobilized Enzymes Catalyzed Reaction Condition}

Given the results of Rh2 synthesized by free enzymes M315F-AtSuSy coupled reaction in our previous report [21], some reaction conditions for the co-immobilized enzymes catalyzed reactions were the same as free enzymes. The reaction contained sucrose $(0.4 \mathrm{M})$ and $\mathrm{MgSO}_{4}(5 \mathrm{mM})$, Tris- $\mathrm{HCl}$ buffer ( $\left.\mathrm{pH} 8.0\right)$, and was conducted at a temperature of $35^{\circ} \mathrm{C}$ and $200 \mathrm{rpm}$.

Additionally, other conditions of the co-immobilized M315F-AtSuSy catalyzed transglycosylating reaction were further reselected. The effects of DMSO concertation (0-20\%), PPD concentration (2-10 mM), UDP (0.5-2.5 mM), and biocatalyst usage (6-21 U) on the Rh2 biosynthesis were studied, respectively. After reactions were regularly monitored, samples were analyzed by HPLC as in the previous report [21].

\subsection{Reusability of Co-Immobilized Enzymes and Fed-Batch Strategy}

The operational stability for the co-immobilized M315F and AtSuSy coupled reaction was examined by repeated usage of co-immobilized enzymes. At the end of each batch, the immobilized enzymes were removed and washed with fresh buffer ( $\mathrm{pH}$ 8.0), and then added to the initial reaction mixture of the next cycle. The reusability was measured as the yield of Rh2 produced by each time.

For the co-immobilized M315F and AtSuSy coupled reaction $(20 \mathrm{~mL})$ with fed-batch method, the optimal conditions of initial concentration of PPD (8 mM), UDP (0.5 mM), sucrose (0.4 M), 50 mM Tris- $\mathrm{HCl}(\mathrm{pH} 8.0), 10 \%(v / v)$ DMSO and $\mathrm{MgSO}_{4}(5 \mathrm{mM})$ in Tris$\mathrm{HCl}$ buffer ( $\mathrm{pH} 8.0$ ), and conducted at a temperature of $35^{\circ} \mathrm{C}$ and $200 \mathrm{rpm}$. During the proceed reaction, the sample was monitored by HPLC [21], fresh PPD $(0.2 \mathrm{~mL})$ dissolved in DMSO (100 mM) was stepwise added to the reactants at 2, 4, 6, 8,10, and $12 \mathrm{~h}$, and a total concentration of PPD was added to the final reaction mixture. 


\section{Conclusions}

In summary, glycosyltransferase Bs-YjiC mutant M315F and sucrose synthase AtSuSy was exploited to biosynthesize rare ginsenoside Rh2 in one-pot reaction from UDP and sucrose. This study disclosed that resin LX1000HG modified with glyoxyl-metal-chelate bifunctional groups were useful for co-immobilization of the engineered enzymes with his-tags. Using the two-step process of specific adsorption and multipoint covalent attachment, the co-immobilized enzymes showed the advantages of improved binding stability, improved $\mathrm{pH}$ and thermal stabilities, and good operation stability. Interestingly, a higher yield was achieved using co-immobilized enzymes through allowing the bioreaction at a high initial concentration of PPD by alleviating inhibition, and the noticeable titer of ginsenoside Rh2 reached the high level of $10.3 \mathrm{~g} / \mathrm{L}$ by periodic feeding of PPD. This study established a green and sustainable approach for the production of ginsenoside Rh2, and it may provide promising candidates for natural drug research and development.

\section{Patents}

There are no patents resulting from the work reported in this manuscript.

Supplementary Materials: The following are available online at https://www.mdpi.com/2073-4 344/11/1/132/s1, Figure S1: Optimization of the adsorbed condition for enzymes of M315F and AtSuSy bound, Figure S2: FTIR spectra of resin LX1000HG, LX1000HG-glyoxyl- $\mathrm{Ni}^{2+}$ and LX1000HGglyoxyl- $\mathrm{Ni}^{2+}$ with enzymes, Figure S3: Effect of $\mathrm{pH}$ on the activities of free and immobilized enzyme M315F/AtSuSy, Figure S4: pH stability of free and immobilized enzyme M315F/AtSuSy, Figure S5: Effect of temperature on the activities of free and immobilized enzyme M315F/AtSuSy, Figure S6: Effect of UDP concentration on the immobilized M315F and AtSuSy coupled reactions for Rh2 synthesis, Figure S7: Activation procedure for heterofunctional resin preparation.

Author Contributions: J.C., methodology, data curation, and writing-original draft. J.Y., methodology, investigation, formal analysis. Y.L., investigation, enzyme immobilization, and conditions optimization. S.Q., conceptualization, validation, data analysis. B.W., conceptualization, supervision, writing-review \& editing. B.H. conceptualization, supervision, visualization, writing-review \& editing. All authors have read and agreed to the published version of the manuscript.

Funding: This research was supported by the National Key Research and Development Program of China (2018YFA0902000), the National Natural Science Foundation of China $(21776135,81673321)$, Primary Research \&Development Plan of Jiangsu (BE2018395), Natural Science Foundation of Jiangsu province (Grant No. BK20181379).

Institutional Review Board Statement: Not applicable.

Informed Consent Statement: Not applicable.

Data Availability Statement: Data is contained within the article.

Acknowledgments: We are grateful to the High Performance Computing Center of Nanjing Tech University for supporting the computational resources.

Conflicts of Interest: The authors declare no conflict of interest.

\section{References}

1. Liu, Z.Q. Chemical Insights into Ginseng as a Resource for Natural Antioxidants. Chem. Rev. 2012, 112, 3329-3355. [CrossRef] [PubMed]

2. Christensen, L.P.; Jensen, M. Biomass and content of ginsenosides and polyacetylenes in American ginseng roots can be in-creased without affecting the profile of bioactive compounds. J. Nat. Med. 2009, 63, 159-168. [CrossRef] [PubMed]

3. Wong, A.S.T.; Che, C.-M.; Leung, K.-W. Recent advances in ginseng as cancer therapeutics: A functional and mechanistic overview. Nat. Prod. Rep. 2015, 32, 256-272. [CrossRef] [PubMed]

4. Li, B.; Zhao, J.; Wang, C.-Z.; Searle, J.S.; He, T.-C.; Yuan, C.-S.; Du, W. Ginsenoside Rh2 induces apoptosis and paraptosis-like cell death in colorectal cancer cells through activation of p53. Cancer Lett. 2011, 301, 185-192. [CrossRef] [PubMed]

5. Kim, H.-S.; Lee, E.-H.; Ko, S.-R.; Choi, K.-J.; Park, J.-H.; Im, D.-S. Effects of ginsenosides Rg3 and Rh2 on the proliferation of prostate cancer cells. Arch. Pharmacal Res. 2004, 27, 429-435. [CrossRef] [PubMed]

6. Nakata, H.; Kikuchi, Y.; Tode, T.; Hirata, J.; Kita, T.; Ishii, K.; Kudoh, K.; Nagata, I.; Shinomiya, N. Inhibitory effects of ginsenoside Rh-2 on tumor growth in nude mice bearing human ovarian cancer cells. Jpn. J. Cancer Res. 1998, 89, 733-740. [CrossRef] [PubMed] 
7. Kim, H.-E.; Oh, J.H.; Lee, S.K.; Oh, Y.J. Ginsenoside RH-2 induces apoptotic cell death in rat C6 glioma via a reactive oxygen- and caspase-dependent but Bcl-XL-independent pathway. Life Sci. 1999, 65, PL33-PL40. [CrossRef]

8. Shibata, S. Chemistry and Cancer Preventing Activities of Ginseng Saponins and Some Related Triterpenoid Compounds. J. Korean Med. Sci. 2001, 16, S28-S37. [CrossRef]

9. Kamra, P.; Gokhale, R.S.; Mohanty, D. SEARCHGTr: A program for analysis of glycosyltransferases involved in glycosylation of secondary metabolites. Nucleic Acids Res. 2005, 33, W220-W225. [CrossRef]

10. Wang, P.P.; Wei, Y.J.; Fan, Y.; Liu, Q.F.; Wei, W.; Yang, C.S.; Zhang, L.; Zhao, G.P.; Yue, J.M. Production of bioactive gin-senosides Rh2 and Rg3 by metabolically engineered yeasts. Metab. Eng. 2015, 29, 97-105. [CrossRef]

11. Hu, Z.-F.; Gu, A.-D.; Liang, L.; Li, Y.; Gong, T.; Chen, J.-J.; Chen, T.-J.; Yang, J.-L.; Zhu, P. Construction and optimization of microbial cell factories for sustainable production of bioactive dammarenediol-II glucosides. Green Chem. 2019, 21, 3286-3299. [CrossRef]

12. Jung, S.-C.; Kim, W.; Park, S.C.; Jeong, J.; Park, M.K.; Lim, S.; Lee, Y.; Im, W.-T.; Lee, J.H.; Choi, G.; et al. Two Ginseng UDP-Glycosyltransferases Synthesize Ginsenoside Rg3 and Rd. Plant Cell Physiol. 2014, 55, 2177-2188. [CrossRef] [PubMed]

13. Wang, P.; Wei, W.; Ye, W.; Li, X.; Zhao, W.; Yang, C.; Li, C.; Yan, X.; Zhou, Z. Synthesizing ginsenoside Rh2 in Saccharomyces cerevisiae cell factory at high-efficiency. Cell Discov. 2019, 5, 1-14. [CrossRef] [PubMed]

14. Hu, Y.M.; Xue, J.; Min, J.; Qin, L.J.; Zhang, J.K.; Dai, L.H. Biocatalytic synthesis of ginsenoside Rh2 using Arabidopsis thaliana glucosyltransferase-catalyzed coupled reactions. J. Biotechnol. 2020, 309, 107-112. [CrossRef] [PubMed]

15. Naoumkina, M.A.; Modolo, L.V.; Huhman, D.V.; Urbanczyk-Wochniak, E.; Tang, Y.; Sumner, L.W.; Dixon, R.A. Genomic and Coexpression Analyses Predict Multiple Genes Involved in Triterpene Saponin Biosynthesis in Medicago truncatula. Plant Cell 2010, 22, 850-866. [CrossRef]

16. Augustin, J.M.; Drok, S.; Shinoda, T.; Sanmiya, K.; Nielsen, J.K.; Khakimov, B.; Olsen, C.E.; Hansen, E.H.; Kuzina, V.; Ekstrøm, C.T.; et al. UDP-Glycosyltransferases from the UGT73C Subfamily in Barbarea vulgaris Catalyze Sapogenin 3-O-Glucosylation in Saponin-Mediated Insect Resistance. Plant Physiol. 2012, 160, 1881-1895. [CrossRef]

17. Zhuang, Y.; Yang, G.-Y.; Chen, X.; Liu, Q.; Zhang, X.; Deng, Z.; Feng, Y. Biosynthesis of plant-derived ginsenoside Rh2 in yeast via repurposing a key promiscuous microbial enzyme. Metab. Eng. 2017, 42, 25-32. [CrossRef]

18. Liang, H.C.; Hu, Z.F.; Zhang, T.T.; Gong, T.; Chen, J.J.; Zhu, P.; Li, Y.; Yang, J.L. Production of a bioactive unnatural gin-senoside by metabolically engineered yeasts based on a new UDP-glycosyltransferase from Bacillus subtilis. Metab. Eng. 2017, 44, 60-69. [CrossRef]

19. Dai, L.; Liu, C.; Li, J.; Dong, C.; Yang, J.; Dai, Z.; Zhang, X.; Sun, Y. One-Pot Synthesis of Ginsenoside Rh2 and Bioactive Unnatural Ginsenoside by Coupling Promiscuous Glycosyltransferase from Bacillus subtilis 168 to Sucrose Synthase. J. Agric. Food Chem. 2018, 66, 2830-2837. [CrossRef]

20. Dai, L.H.; Li, J.; Yang, J.G.; Zhu, Y.M.; Men, Y.; Zeng, Y.; Cai, Y.; Dong, C.X.; Dai, Z.B.; Zhang, X.L.; et al. Use of a Pro-miscuous Glycosyltransferase from Bacillus subtilis 168 for the Enzymatic Synthesis of Novel Protopanaxatriol-Type Ginseno-sides. J. Agric. Food Chem. 2018, 66, 943-949. [CrossRef]

21. Ma, W.; Zhao, L.; Ma, Y.; Li, Y.; Qin, S.; He, B. Oriented efficient biosynthesis of rare ginsenoside Rh2 from PPD by compiling UGT-Yjic mutant with sucrose synthase. Int. J. Biol. Macromol. 2020, 146, 853-859. [CrossRef] [PubMed]

22. Cao, L.Q. Immobilised enzymes: Science or art? Curr. Opin. Chem. Biol. 2005, 9, 217-226. [CrossRef] [PubMed]

23. Sheldon, R.A.; van Pelt, S. Enzyme immobilisation in biocatalysis: Why, what and how. Chem. Soc. Rev. 2013, 42, 6223-6235. [CrossRef] [PubMed]

24. Pescador, P.; Katakis, I.; Toca-Herrera, J.L.; Donath, E. Efficiency of a Bienzyme Sequential Reaction System Immobilized on Polyelectrolyte Multilayer-Coated Colloids. Langmuir 2008, 24, 14108-14114. [CrossRef]

25. Feng, J.; Balaji, N.; Surya, M. Materials-Based Strategies for Multi-Enzyme Immobilization and Co-Localization: A Review. Biotechnol. Bioeng. 2014, 111, 209-222. [CrossRef]

26. Ley, C.; Holtmann, D.; Mangold, K.-M.; Schrader, J. Immobilization of histidine-tagged proteins on electrodes. Colloids Surf. B Biointerfaces 2011, 88, 539-551. [CrossRef]

27. Liu, Z.; Zhang, J.; Chen, X.; Wang, P.G. Combined Biosynthetic Pathway for De Novo Production of UDP-Galactose: Catalysis with Multiple Enzymes Immobilized on Agarose Beads. ChemBioChem 2002, 3, 348-355. [CrossRef]

28. Kim, H.; Kwon, H.-S.; Ahn, J.; Lee, C.-H.; Ahn, I.-S. Evaluation of a silica-coated magnetic nanoparticle for the immobilization of a His-tagged lipase. Biocatal. Biotransformation 2009, 27, 246-253. [CrossRef]

29. de Melo, R.R.; Alnoch, R.C.; Vilela AF, L.; de Souza, E.M.; Krieger, N.; Ruller, R.; Sato, H.H.; Mateo, C. New Heterofunctional Supports Based on nanoparticle for the immobilization: A Tool for Enzyme Immobilization at Neutral pH. Molecules 2017, 22, 1088. [CrossRef]

30. Li, X.; Huang, S.S.; Xu, L.; Yan, Y.J. Improving activity and enantioselectivity of lipase via immobilization on macroporous resin for resolution of racemic 1-phenylethanol in non-aqueous medium. BMC Biotechnol. 2013, 13, 92. [CrossRef]

31. Blanco, R.M.; Terreros, P.; Muñoz, N.; Serra, E. Ethanol improves lipase immobilization on a hydrophobic support. J. Mol. Catal. B Enzym. 2007, 47, 13-20. [CrossRef]

32. Mateo, C.; Bolívar, J.M.B.; Godoy, C.A.; Rocha-Martin, J.; Pessela, B.C.; Curiel, J.A.; Muñoz, R.; Guisán, J.M.; FernándezLorente, G. Improvement of Enzyme Properties with a Two-Step Immobilization Process on Novel Heterofunctional Supports. Biomacromolecules 2010, 11, 3112-3117. [CrossRef] [PubMed] 
33. Mateo, C.; Abian, O.; Fernández-Lorente, G.; Pedroche, J.; Fernández-Lafuente, R.; Guisan, J.M.; Tam, A.; Daminati, M. Epoxy Sepabeads: A Novel Epoxy Support for Stabilization of Industrial Enzymes via Very Intense Multipoint Covalent Attachment. Biotechnol. Prog. 2002, 18, 629-634. [CrossRef] [PubMed]

34. Mokhtar, N.F.; Rahman, R.N.Z.R.A.; Noor, N.D.M.; Shariff, F.M.; Ali, M.S.M. The Immobilization of Lipases on Porous Support by Adsorption and Hydrophobic Interaction Method. Catalysts 2020, 10, 744. [CrossRef]

35. Bradford, M.M. A rapid and sensitive method for the quantitation of microgram quantities of protein utilizing the principle of protein-dye binding. Anal. Biochem. 1976, 72, 248-254. [CrossRef] 